\title{
Tuberculosis control in South Africa: Successes, challenges and recommendations
}

G J Churchyard ${ }^{1-3}$ MB BCh, PhD; L D Mametja, ${ }^{4}$ MPH (Health Pol \& Manag); L Mvusi, ${ }^{4}$ MB BCh; N Ndjeka, ${ }^{4}$ MB BCh, MMed (Fam Med); A C Hesseling, ${ }^{5} \mathrm{MB} \mathrm{ChB}, \mathrm{MSc}, \mathrm{PhD}$; A Reid, ${ }^{6} \mathrm{MB} \mathrm{ChB}, \mathrm{MD}, \mathrm{MPH}$; S Babatunde, ${ }^{7} \mathrm{MB} \mathrm{BS}, \mathrm{MPH}$; Y Pillay, ${ }^{4} \mathrm{PhD}$

${ }^{1}$ Aurum Institute, Johannesburg, South Africa

${ }^{2}$ School of Public Health, Faculty of Health Sciences, University of the Witwatersrand, Johannesburg, South Africa

${ }^{3}$ London School of Hygiene and Tropical Medicine, London, UK

${ }^{4}$ National Department of Health, Pretoria, South Africa

${ }^{5}$ Desmond Tutu TB Centre, Department of Paediatrics and Child Health, Faculty of Health Sciences, Stellenbosch University, Tygerberg, South Africa

${ }^{6}$ UNAIDS, South Africa

World Health Organization, South Africa

Corresponding author: G J Churchyard (gchurchyard@auruminstitute.org)

Tuberculosis (TB) remains a global health threat, and South Africa (SA) has one of the world's worst TB epidemics driven by HIV. Among the 22 countries with the highest burden of TB, SA has the highest estimated incidence and prevalence of TB, the second highest number of diagnosed multidrug-resistant TB cases, and the largest number of HIV-associated TB cases. Although SA has made notable progress in reducing TB prevalence and deaths and improving treatment outcomes for new smear-positive TB cases, the burden of TB remains enormous. SA has the means to overcome this situation. In addition to better implementing the basics of TB diagnosis and treatment, scaling up the use of Xpert MTB/RIF as a replacement for sputum smear microscopy, strengthening case finding in and beyond healthcare facilities and a greater focus on TB prevention for people living with HIV, particularly earlier initiation of and scaling up antiretroviral therapy and scaling up continuous isoniazid preventive therapy, will have a substantial impact on TB control. New TB drugs, diagnostics and vaccines are required to further accelerate progress towards improved TB control in SA and beyond.

S Afr Med J 2014;104(3 Suppl 1):244-248. DOI:10.7196/SAMJ.7689

口斗回

Tuberculosis (TB) remains a global health threat. Despite a slow decline in global TB rates, the World Health Organization (WHO) reported 8.6 million new cases of TB in 2012 (13\% in people living with HIV), resulting in 1.3 million deaths $(23 \%$ among 口. HIV-positive persons). ${ }^{[1]}$ South Africa (SA) has one of the world's worst TB epidemics driven by HIV. The WHO has identified 22 high-burden countries (HBCs), which account for $81 \%$ of all estimated incident $\mathrm{TB}$ cases globally.

Among the HBCs, SA has the third highest absolute number of reported incident cases and the fifth highest number of estimated prevalent (undiagnosed active TB) cases. ${ }^{[1]}$ After adjusting for population size, SA has the highest incidence and prevalence of TB among the HBCs. It also has the largest number of HIV-associated TB cases and the second-largest number of diagnosed multidrugresistant (MDR)-TB cases (after India). ${ }^{[1]}$

The National Tuberculosis Programme (NTP), established after SA became a democracy in 1994, faced the challenge of integrating TB services into weak primary healthcare systems and the emergence of the HIV epidemic, which led to TB case rates quadrupling between 1994 and 2012 (Fig. 1). ${ }^{[2]}$ The growing burden of MDR-TB and the emergence of extensively drug-resistant (XDR) TB in 2006 added a further burden to overstretched health services. In order to respond to the dual epidemics of HIV and TB rationally, SA developed an integrated National Strategic Plan (NSP) for HIV, STIs and TB $(2012-2016) .{ }^{[3]}$ The targets set in the NSP for TB are to halve TB incidence and mortality by 2016 and to have no new TB infections, deaths or stigma by 2032. In this review we highlight successes and challenges in TB prevention, treatment and care and discuss strategies to achieve the NSP targets.

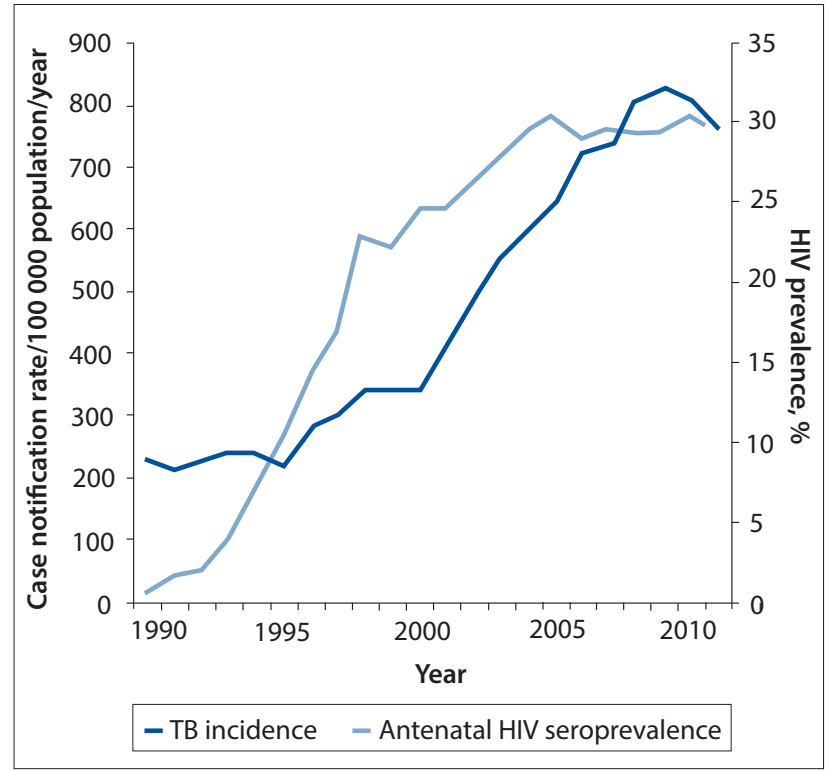

Fig. 1. Trends in tuberculosis case notification rates and HIV prevalence in South Africa. 


\section{TB control}

The central pillars of $\mathrm{TB}$ control include finding, treating and preventing TB in order to avoid TB deaths and reduce transmission. The NTP has substantially strengthened the TB control programme since 1994, and the major milestones are summarised in Table 1. Encouragingly, the efforts of the NTP have contributed to a slow decline in TB case notification rates since 2009 (Fig. 1).

\section{Find TB}

SA has an extensive network of microscopy centres and diagnostic laboratories with the capacity for culture and drug susceptibility testing using the Mycobacterial Growth Indicator Tube liquid culture system. In 2011, SA introduced Xpert MTB/RIF as a replacement for sputum smear microscopy for the diagnosis of pulmonary TB. Between March 2011 and April 2013 >1.3 million Xpert MTB/RIF tests were done in SA, which accounted for more than half of the global usage of Xpert MTB/RIF. SA also pioneered the use of dried culture spots for verification and external quality assurance of Xpert $\mathrm{MTB} / \mathrm{RIF}^{[4]}$ and implemented a central data monitoring system to remotely monitor the performance of Xpert MTB/RIF, the proportion of positive tests and the proportion with rifampicin resistance.

Although nationwide implementation of Xpert MTB/RIF is almost complete, numerous challenges to its implementation remain. Xpert is not universally utilised and there has been poor adherence to the Xpert algorithm, requiring simplification of the algorithm for monitoring treatment response and investigation of HIV-positive, Xpert-negative persons with suspected TB. Ensuring an uninterrupted supply of cartridges will remain a challenge as the global demand for Xpert cartridges increases. The recording and reporting of Xpert results remain suboptimal.

\section{Treat TB}

SA's treatment success rate among new smear-positive and smearnegative/extrapulmonary TB patients has improved to $79 \%$ and $76 \%$, respectively, achieved largely as a result of an increase in cure rates and a decline in the treatment default rate following the introduction of community-based tracing teams. ${ }^{[1]}$ The treatment success rate among retreatment cases remains poor at $66.3 \%$. Of concern, up to $25 \%$ of sputum smear-positive TB cases are lost to follow-up before treatment initiation, which may contribute to ongoing transmission and an increased risk of death. ${ }^{[5]}$ Strategies to reduce initial loss to follow-up are required. The mortality rate remains high even after completion of TB treatment, probably due to HIV disease. ${ }^{[6]}$ Scaling up antiretroviral therapy (ART) to all HIV-infected TB patients is required to reduce HIV-related mortality among TB patients.

The national rollout of Xpert MTB/RIF has enabled rapid diagnosis of rifampicin resistance. As a result $\mathrm{SA}$ is in the process of withdrawing the use of streptomycin for retreatment TB: rifampicinsusceptible retreatment $\mathrm{TB}$ cases are treated with a first-line regimen, and rifampicin-resistant retreatment cases are managed according to MDR-TB guidelines.

\section{Prevent TB}

Prevention of TB has been a neglected aspect of TB control. TB prevention strategies include: treatment of latent TB infection among high-risk persons; case finding to detect and treat infectious TB earlier, reducing the duration of infectiousness and transmission; early ART for people living with HIV; and TB vaccination strategies. In this regard, SA adopted the '3Is' policy of isoniazid preventive therapy (IPT), intensified case finding and infection control in 2002.

\begin{tabular}{|c|c|}
\hline 1997 & Phased implementation of DOTS, establishment of DTDs \\
\hline 1999 & $\begin{array}{l}\text { Introduction of fixed-dose combination drugs } \\
\text { Establishment of TB and HIV pilot districts }\end{array}$ \\
\hline 2000 & $\begin{array}{l}\text { MDR-TB guidelines endorsed, establishment of MDR-TB } \\
\text { treatment facilities } \\
\text { Four-drug fixed-dose combination tablets introduced }\end{array}$ \\
\hline 2001 & National Drug Resistance Survey \\
\hline 2002 & $\begin{array}{l}\text { Launch of the MTDP, } 2002 \text { - } 2005 \\
\text { Guidelines for IPT for TST-positive, HIV-infected persons }\end{array}$ \\
\hline 2003 & $\begin{array}{l}\mathrm{TB} \text { declared an emergency and } \mathrm{TB} \text { crisis plan launched } \\
\text { Electronic } \mathrm{TB} \text { register introduced }\end{array}$ \\
\hline 2005 & $\begin{array}{l}\text { Minister of Health signs 'Declaration of TB as an } \\
\text { emergency in AFRO region' }\end{array}$ \\
\hline 2006 & Development of MDR-TB and XDR-TB action plan \\
\hline 2007 & $\begin{array}{l}\text { Launch of the National TB Strategic Plan } 2007 \text { - } 2011 \\
\text { Development of infection control guidelines for TB }\end{array}$ \\
\hline 2008 & $\begin{array}{l}\text { Introduction of Hain MTBDRplus as a rapid test for } \\
\text { MDR-TB } \\
\text { First SA TB conference }\end{array}$ \\
\hline 2009 & $\begin{array}{l}\text { 'Health in South Africa' series published in The Lancet, } \\
\text { including recommendations for TB/HIV } \\
\text { WHO review of the NTP }\end{array}$ \\
\hline 2010 & $\begin{array}{l}\text { 6-month IPT for all HIV-infected persons, regardless of } \\
\text { TST status } \\
\text { ART for TB patients living with HIV with } \mathrm{CD} 4^{+} \text {counts } \\
<350 \text { cells } / \mu \text { l }\end{array}$ \\
\hline 2011 & $\begin{array}{l}\text { Introduction of Xpert MTB/Rif as a replacement for } \\
\text { sputum smear microscopy } \\
\text { National HIV/TB campaign } \\
\text { Management of DR TB policy guidelines approved } \\
\text { Decentralised management of MDR-TB introduced }\end{array}$ \\
\hline 2012 & $\begin{array}{l}\text { SA President signs SADC declaration on 'TB in the mines' } \\
\text { ART for all HIV-infected TB patients } \\
\text { Streptomycin removed from retreatment regimen }\end{array}$ \\
\hline 2013 & $\begin{array}{l}\text { NDoH guidelines for managing TB/HIV in prisons issued } \\
\text { IPT for at least } 36 \text { months for TST-positive, HIV-infected } \\
\text { persons } \\
\text { National drug resistance survey } \\
\text { Independent WHO Review of NTP }\end{array}$ \\
\hline \multicolumn{2}{|c|}{ 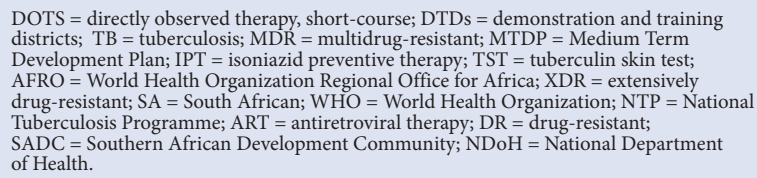 } \\
\hline
\end{tabular}

\section{TB screening}

TB screening of high-risk persons or groups may contribute to reduced deaths and TB transmission. The WHO recommends that people living with HIV are systematically screened for TB at each contact with the heath service, using a symptom screen. In 2012, 949800 HIV-positive South Africans were screened for TB, which although impressive is still substantially below the total number of people living with HIV in care. ${ }^{[1]}$ Among persons on long-term ART, 
the prevalence of undiagnosed $\mathrm{TB}$ remains high, and underscores the need for ongoing TB screening. ${ }^{[7,8]}$ TB screening should be scaled up to achieve high coverage to ensure that HIV-infected persons with TB are diagnosed and treated appropriately.

SA introduced a multifaceted TB screening programme in 2011, focused on high-burden districts, which included household contact tracing, HIV counselling and testing campaigns, community mobilisation, door-to-door enquiry in areas with a high burden of smear-positive TB, and screening of high-risk populations. During $2011>150000$ household contacts were screened for TB and $>3000$ new cases, which would not have been detected through routine means, were identified (Lo T, 'Rapid assessment of intensified tuberculosis case finding efforts, Republic of South Africa' unpublished report by the US Centers for Disease Control to the National Department of Health (NDoH), 20 March 2012, available on request from Mr Lerole Mametja, Chief Director of the National TB Control and Management Cluster). This report also states that challenges to the sustained implementation of household-based case finding include funding, human resource constraints and lack of customised recording and reporting tools.

IPT

The IPT guidelines for tuberculin skin test (TST)-positive people living with HIV were initially incorporated into the ART guidelines. Uptake of IPT was poor following introduction of these guidelines, largely due to the TST creating a barrier to implementation and concerns of generating isoniazid resistance. In 2010, the IPT guidelines were revised to remove the TST to facilitate programmatic implementation of IPT. The uptake of IPT increased dramatically and in 2011 and 2012, >375 000 South Africans living with HIV initiated IPT, making SA's IPT programme one of the largest in the world. Despite this, the uptake of IPT among people living with HIV in care, including children and pregnant women, remains poor. ${ }^{[9-11]}$ The IPT guidelines were re-issued in 2013 as part of the revised ART guidelines to recommend at least 36 months of IPT to TSTpositive persons or 6 months of IPT for those whose TST status is unknown. ${ }^{[12-15]}$ Stand-alone IPT guidelines are being developed for adults and children living with HIV and persons with silicosis. The reintroduction of TST into the IPT guidelines may pose a barrier to implementation of continuous IPT.

\section{Infection control}

People living with HIV and young children are at a high risk of exposure to drug-susceptible and -resistant $\mathrm{TB}$ when attending health facilities for care. Furthermore, healthcare workers are also at high risk of acquiring TB nosocomially, which highlights the importance of stringent implementation of infection control policies in order to reduce TB transmission of drug-susceptible and -resistant $\mathrm{TB}$ in healthcare facilities and other congregate settings. ${ }^{[16]} \mathrm{SA}$ introduced a TB infection control programme in 2007 , which included a structural review of healthcare facilities and process re-engineering if required. Minimum standards for health facilities have been developed by the Council for Scientific and Industrial Research. However, the implementation of infection control guidelines remains sub-optimal. Healthcare facilities should be monitored regularly to ensure consistent implementation of infection control guidelines. Infection control in other congregate settings, such as correctional service facilities, community halls, schools and public transportation (taxis and buses), is also required.
ART

ART reduces the TB risk by $65 \%$ overall, and across all $\mathrm{CD} 4^{+}$strata, suggesting that earlier ART initiation may contribute to national TB control activities. ${ }^{[17]}$ SA has the largest ART programme in the world. Although ART substantially reduces the risk of TB among people living with HIV, the rate of TB remains four-fold higher than among HIVnegative persons from the same community. ${ }^{[18]}$ IPT given with ART is safe and further reduces the risk of TB by $37 \%$, regardless of TST status. ${ }^{[19]}$ SA should therefore scale up the provision of IPT to patients on ART. In order for the ART programme to contribute maximally to TB control, SA should strive to achieve high coverage of ART and consider initiating ART even earlier, including in pregnant women. ${ }^{[1,20]}$

\section{MDR-TB}

The number of MDR-TB and XDR-TB cases in SA has increased substantially, with 15419 and 1596 cases, respectively, diagnosed in 2012. ${ }^{[1]}$ The treatment success rate for adult MDR- and XDR-TB remains low at $42 \%$ and $18 \%$, respectively, for the 2010 cohort. The MDR-TB sub-programme has faced many challenges, including confirmation of MDR-TB by mycobacterial growth inhibition tube (MGIT) liquid culture drug susceptibility testing in only a third of patients; high initial loss to follow-up of MDR-TB patients, with approximately $40 \%$ of patients not initiating appropriate treatment; inadequate bed capacity; poor infection control; limited availability of appropriate second-line drugs and paediatric formulations; and poor adherence to treatment ( $\mathrm{N}$ Ndjeka, $\mathrm{NDoH}$ - personal communication). In response to the large burden of MDR-TB, the NTP committed $>\$ 200$ million to the MDR-TB programme; issued a series of guidelines for the management of MDR-TB; established 45, mainly decentralised, MDR- and XDR-TB treatment units, with approximately 2 500-bed capacity; and introduced a policy framework for the decentralisation and de-institutionalisation of drug-resistant TB care and treatment. ${ }^{[2,22]}$ The current standardised adult MDR-TB treatment regimen is associated with poor cure rates. In order to improve cure rates of MDR-TB, clofazimine has been made available, the role of linezolid is being considered, and a bedaquiline access programme has been introduced.

\section{Maternal and childhood TB}

Childhood TB accounted for $13 \%$ of all new TB cases in 2012. ${ }^{[23]}$ Treatment outcomes for childhood TB are relatively good, with $82 \%$ of cases successfully treated in 2010 . SA enjoys a strong political commitment for addressing the challenges of childhood TB; a blueprint for action on paediatric and adolescent HIV and TB prevention, treatment, care and support has been developed and implemented. South Africans have played a leading role in revising the 2010 WHO childhood TB treatment guidelines, ${ }^{[2]}$ which were adopted in SA. Notable advances in childhood TB include changing the age and disease categories for reporting, simplifying contact management of TB-exposed children and children living with HIV; revision of the Integrated Management of Childhood Illness guidelines to include TB screening and management; and scaling up prevention of mother-to-child transmission of HIV programmes. Many challenges persist for management of childhood $\mathrm{TB}$, including the lack of fixed-dose combinations for first-line drugs; limited child-friendly second-line drug formulations; poor implementation of IPT in HIV-infected child contacts; gaps in recording and reporting of childhood TB; weak health systems to manage paediatric TB; and poor linkage to care, particularly from the hospital to the community. 


\section{TB/HIV integration}

$\mathrm{TB} / \mathrm{HIV}$ integration requires activities to reduce the burden of TB in people living with HIV (discussed above) and to reduce the burden of HIV in TB patients. Initiating ART while on TB treatment saves lives, regardless of $\mathrm{CD} 4^{+}$count, and among persons with $\mathrm{CD} 4^{+}$counts $<50$ cells/ $\mu$ l, initiating ART soon after initiating TB treatment is essential to reduce mortality. ${ }^{[6,25]}$ Decentralisation of ART services to primary healthcare clinics and integration with $\mathrm{TB}$ services have resulted in increasing numbers of TB patients initiating ART. In 2012, only $54 \%$ of HIV-positive TB patients were initiated on ART, ${ }^{[1]}$ highlighting the need to scale up ART for TB patients further by scaling up nurse-initiated and managed ART training for all professional nurses.

SA has played a leading role in evaluating strategies to integrate TB and HIV services. ${ }^{[26]}$ The PALSA PLUS intervention, which promotes integration by providing on-site, case-based, iterative education led by a trainer, increased the yield of TB diagnoses. ${ }^{[27]}$ Guidelines for integrating TB and HIV services at primary healthcare facilities were developed and disseminated in 2010, but TB/HIV integration remains poorly implemented and strategies to strengthen integration of services, including ART, are required. ${ }^{[28,2]}$

\section{Healthcare systems}

The SA government is strengthening the healthcare system through a process of primary healthcare re-engineering to balance communitybased preventive services with hospital-based curative services, and phased implementation of a National Health Insurance to ensure affordability, effectiveness and universal access to quality healthcare services, including TB diagnostic and treatment services. ${ }^{[30]}$ These initiatives aim to support the implementation of TB control activities and promote integration with other primary healthcare services, including child and maternal healthcare programmes. Addressing the challenge of poor health service management will, however, be critical for effective implementation of these government initiatives.

\section{Surveillance and information}

The NTP has developed an electronic recording and reporting system for drug-susceptible TB (ETR.net) and drug-resistant TB (EDR. net). There are, however, numerous challenges with the electronic recording and reporting systems, including inadequate numbers and high turnover of data capturers and poor-quality data entry; linking between these two systems; ensuring that hospital-diagnosed cases are captured adequately; multiple programme registers and repeated data entry at facility level; and lack of a unique health identifier to track patients who move between districts and across programmes. Novel and sustainable solutions, such as the use of mobile phones and tablets to collect data in the field and in facilities, are required to strengthen the quality and use of data as well as patient management.

The performance of the NTP is routinely monitored by drug resistance and TB prevalence surveys and independent programme reviews. The NTP is currently conducting a national drug resistance survey, the first national TB prevalence survey is planned, and an independent review of the NTP has recently been completed. The NTP is also currently implementing the WHO TB Surveillance Checklist to assess the effectiveness of the various TB surveillance systems in estimating the burden of TB.

\section{Key populations}

TB has been a major health threat in the SA mining industry since its inception in the late 1800s. SA goldminers have one of the highest $\mathrm{TB}$ incidence rates in the world, currently estimated at $3000 / 100000$ population. ${ }^{[14]}$ To address the TB epidemic in the mines, the SA
Minister of Health, Dr Aaron Motsoaledi, along with the ministers of health of Lesotho and Swaziland, spearheaded the development of a Southern African Development Community declaration signed by the heads of state that provides a framework for addressing TB in the mines at a regional level. This constitutes the first ever comprehensive multidisciplinary regional response to $\mathrm{TB}$ in the mines.

$\mathrm{TB}$ is a major health problem in SA prisons due to overcrowding. The prevalence of undiagnosed active TB is high $(2.4 \%),{ }^{[31]}$ and requires effective case-finding strategies linked to early treatment initiation to ensure cure. The $\mathrm{NDoH}$ and the Department of Correctional Services have issued TB and HIV guidelines to ensure effective control of TB in prisons. Implementation and monitoring and evaluation of these guidelines will be critically important.

\section{Strategies to achieve the NSP targets}

Additional strategies are required to meet the NSP and Stop TB partnership targets. Mathematical modelling suggests that in addition to doing the basics better, scaling up ART and initiating ART earlier, scaling up use of Xpert MTB/RIF as a replacement for sputum smear microscopy, strengthening targeted case finding in healthcare facilities and communities, and introducing and scaling up continuous IPT will have a substantial impact on TB control. However, to achieve the NSP targets, new effective TB regimens, diagnostics and vaccines need to be developed, with consideration of adults and children living with $\mathrm{HIV}^{[32]}$

\section{Conclusion}

SA has made notable progress in improving TB control, but the burden of TB remains enormous. This situation can be reversed. Ensuring high, sustained coverage of effective interventions for TB and HIV, including in children and special populations, will substantially reduce the burden of TB and result in the NSP targets being met. New drugs, diagnostics and vaccines are required to accelerate progress towards TB elimination.

\section{References}

1. World Health Organization. Global Tuberculosis Report 2012. http://www.who.int/tb/publications/ global_report/gtbr12_main.pdf (accessed 21 January 2014).

2. Abdool Karim SS, Churchyard GJ, Karim QA, Lawn SD. HIV infection and tuberculosis in South Africa: An urgent need to escalate the public health response. Lancet 2009;374(9693):921-933. [http:// dx.doi.org/10.1016/S0140-6736(09)60916-8]

3. National Department of Health. National Strategic Plan on HIV, STIs and TB: 2012-2016. http://www doh.gov.za/docs/stratdocs/2012/NSPfull.pdf (accessed 14 October 2013).

4. Scott LE, Gous N, Cunningham BE, et al. Dried culture spots for Xpert MTB/RIF external quality assessment: Results of a phase 1 pilot study in South Africa. J Clin Microbiol 2011;49(12):4356-4360. [http://dx.doi.org/10.1128/JCM.05167-11]

5. Claassens MM, du Toit E, Dunbar R, et al. Tuberculosis patients in primary care do not start treatment: What role do health system delays play? Int J Tuberc Lung Dis 2013;17(5):603-607. [http://dx.doi. org/10.5588/ijtld.12.0505]

org/10.5588/ijtld.12.0505]
6. Abdool Karim SS, Naidoo K, Grobler A, et al. Timing of initiation of antiretroviral drugs during 6. Abdool Karim SS, Naidoo K, Grobler A, et al. Timing of initiation of antiretroviral drugs during
tuberculosis therapy. N Engl J Med 2009;362(8):697-706. [http://dx.doi.org/10.1056/NEJMoa0905848] tuberculosis therapy. N Engl J Med 2009;362(8):697-706. [http://dx.doi.org/10.1056/NEJMoa0905848]
7. Kufa T, Mngomezulu V, Charalambous S, et al. Undiagnosed tuberculosis among HIV clinic attendees: Association with antiretroviral therapy and implications for intensified case finding, isoniazid preventive therapy, and infection control. J Acquir Immune Defic Syndr 2012;60(2):e22-e28. [http:// dx.doi.org/10.1097/QAI.0b013e318251ae0b]

8. Rangaka MX, Wilkinson RJ, Glynn JR, et al. Effect of antiretroviral therapy on the diagnostic accuracy of symptom screening for intensified tuberculosis case finding in a South African HIV clinic. Clin Infect Dis 2012;55(12):1698-1706. [http://dx.doi.org/10.1093/cid/cis775]

9. Van Wyk SS, Mandalakas AM, Enarson DA, Gie RP, Beyers N, Hesseling AC. Tuberculosis contact investigation in a high-burden setting: House or household? Int J Tuberc Lung Dis 2012;16(2):157-162. [http://dx.doi.org/10.5588/ijtld.11.0393]

10. Mandalakas AM, Hesseling AC, Gie RP, Schaaf HS, Marais BJ, Sinanovic E. Modelling the costeffectiveness of strategies to prevent tuberculosis in child contacts in a high-burden setting. Thorax 2013;68(3):247-255. [http://dx.doi.org/10.1136/thoraxjnl-2011-200933]

11. Bekker A, du Preez K, Schaaf HS, Cotton MF, Hesseling AC. High tuberculosis exposure among Bekker A, du Preez K, Schaaf HS, Cotton MF, Hesseling AC. High tuberculosis exposure among
neonates in a high tuberculosis and human immunodeficiency virus burden setting. Int J Tuberc Lung Dis 2012;16(8):1040-1046. [http://dx.doi.org/10.5588/ijtld.11.0821]

12. Samandari T, Agizew TB, Nyirenda S, et al. 6-month versus 36-month isoniazid preventive treatment for tuberculosis in adults with HIV infection in Botswana: A randomised, double-blind, placebo-controlled trial. Lancet 2011;377(9777):1588-1598. [http://dx.doi.org/10.1016/S01406736(11)60204-3]

13. Martinson NA, Barnes GL, Moulton LH, et al. New regimens to prevent tuberculosis in adults with HIV infection. N Engl J Med 2011;365(1):11-20. [http://dx.doi.org/10.1056/NEJMoa1005136]

4. Churchyard GJ, Fielding KL, Lewis JJ, Coetzee L, Godfrey-Faussett P, Hayes RJ. A trial of communitywide isoniazid preventive therapy for tuberculosis control. N Engl J Med 2014;370(4):301-310. [http:// dx.doi.org/10.1056/NEJMoa1214289]

15. National Department of Health. The South African Antiretroviral Treatment Guidelines 2013. http:// www.sanac.org.za/resources/cat_view/1-resources (accessed 14 October 2013). 
16. O'Donnell MR, Jarand J, Loveday M, et al. High incidence of hospital admissions with multidrugresistant and extensively drug-resistant tuberculosis among South African health care workers. Ann Intern Med 2010;153(8):516-522. [http://dx.doi.org/10.7326/0003-4819-153-8-201010190-00008]

17. Suthar AB, Lawn SD, del Amo J, et al. Antiretroviral therapy for prevention of tuberculosis in adults with HIV: A systematic review and meta-analysis. PLoS Med 2012;9(7):e1001270. [http://dx.doi. org/10.1371/journal.pmed.1001270]

18. Gupta A, Wood R, Kaplan R, Bekker LG, Lawn SD. Tuberculosis incidence rates during 8 years of follow-up of an antiretroviral treatment cohort in South Africa: Comparison with rates in the community. PLoS One 2012;7(3):e34156. [http://dx.doi.org/10.1371/journal.pone.0034156]

19. Rangaka MX, Boulle A, Wilkinson RJ, et al. Randomized controlled trial of isoniazid preventive therapy in HIV-infected persons on antiretroviral therapy. Presented at the 19th International AIDS Conference, Washington, DC, USA, $22-27$ July 2012. Abstract THLBB03.

20. Lawn SD, Harries AD, Williams BG et al. Antiretroviral therapy and the control of HIV-associated tuberculosis. Will ART do it? Int J Tuberc Lung Dis 2011;15(5):571-581. [http://dx.doi.org/10.5588/ ijtld.10.0483

21. National Department of Health. Multidrug resistant tuberculosis: A policy framework on decentralised and deinstitutionalised management for South Africa. http://www.doh.gov.za/docs/policy/2011/ policy_TB.pdf (accessed 4 November 2013).

22. National Department of Health. Management of drug-resistant tuberculosis: Policy guidelines. http:// www.tbonline.info/media/uploads/documents/mdr-tb_sa_2010.pdf (accessed 4 November 2013).

23. World Health Organization. Global Tuberculosis Report 2013. Geneva: WHO, 2012, http://www.who. int/tb/publications/global_report/en/index.html (accessed 21 January 2013).

24. World Health Organization. Rapid advice: Treatment of tuberculosis in children. http://whqlibdoc who.int/publications/2010/9789241500449 eng.pdf (accessed 4 November 2013).

25. Abdool Karim SS, Naidoo K, Grobler A, et al. Integration of antiretroviral therapy with tuberculosis treatment. N Engl J Med 2011;365(16):1492-1501. [http://dx.doi.org/10.1056/NEJMoa1014181]

26. Coetzee D, Hilderbrand K, Goemaere E, Matthys F, Boelaert M. Integrating tuberculosis and HIV car Coetzee D, Hilderbrand $\mathrm{K}$, Goemaere E, Matthys F, Boelaert M. Integrating tuberculosis and HIV care
in the primary care setting in South Africa. Trop Med Int Health 2004;9(6):A11-A15. [http://dx.doi. org/10.1111/ji.1365-3156.2004.01259.x

27. Zwarenstein M, Fairall LR, Lombard C, et al. Outreach education for integration of HIV/AIDS care, antiretroviral treatment, and tuberculosis care in primary care clinics in South Africa: PALSA PLU pragmatic cluster randomised trial. BMJ 2011;342:d2022. [http://dx.doi.org/10.1136/bmj.d2022]

28. National Department of Health. A practical guide for TB and HIV service integration at primary health care facilities. http://www.sanacws.org.za/en/resource-centre/download/4ff15962e6d5d-guideon-tb-and-hiv-integration-at-facility-level-pdf (accessed 4 November 2013).

29. Legido-Quigley H, Montgomery CM, Khan P, et al. Integrating tuberculosis and HIV services in lowand middle-income countries: A systematic review. Trop Med Int Health 2013;18(2):199-211. [http:// dx.doi.org/10.1111/tmi.12029]

30. Mayosi BM, Lawn JE, van Niekerk A, Bradshaw D, Abdool Karim SS, Coovadia HM. Health in South Africa: Changes and challenges since 2009. Lancet 2012;380(9858):2029-2043. [http://dx.doi. org/10.1016/S0140-6736(12)61814-5]

31. Telisinghe L, Fielding K, Malden J, et al. Tuberculosis prevalence in a South African prison: Time to implement intensive TB screening. Int J Tuberc Lung Dis 2011;15(Suppl 3):S68.

32. Abu-Raddad LJ, Sabatelli L, Achterberg JT, et al. Epidemiological benefits of more-effective . Abu-Raddad LJ, Sabatelli L, Achterberg JT, et al. Epidemiological benefits of more-effective
tuberculosis vaccines, drugs, and diagnostics. Proc Natl Acad Sci U S A 2009;106(33):13980-13985. tuberculosis vaccines, drugs, and diagnostics.
[http://dx.doi.org/10.1073/pnas.0901720106] 